\title{
Quantum Control and Entanglement using Periodic Driving Fields
}

\author{
C. E. Creffield \\ Department of Physics and Astronomy, University College London, Gower Street, London WC1E 6BT, United Kingdom
} (Received 5 February 2007; published 12 September 2007)

\begin{abstract}
We propose a scheme for producing directed motion in a lattice system by applying a periodic driving potential. By controlling the dynamics by means of the effect known as coherent destruction of tunneling, we demonstrate a novel ratchetlike effect that enables particles to be coherently manipulated and steered without requiring local control. Entanglement between particles can also be controllably generated, which points to the attractive possibility of using this technique for quantum information processing.
\end{abstract}

Introduction. - Controlling the time evolution of quantum states and engineering entanglement between quantum particles are two of the major tasks required for quantum information processing. Although the majority of experimental demonstrations of entanglement distribution have so far employed photons, future practical implementations of quantum computers will almost certainly be based on condensed matter systems. Efforts in this direction include controlling electronic charge or spin ("spintronic") degrees of freedom in coupled quantum dots [1], manipulating the dynamics of Josephson junctions [2], and using spin chains [3] as quantum communication channels. Recently bosons held in optical lattice potentials have also been suggested as possible candidates, using controlled collisions [4] or the dipole-dipole interaction [5] to manipulate the system.

In this work we demonstrate how particles in a lattice potential can be controlled by applying an oscillatory driving field. Control is achieved by using the effect termed "coherent destruction of tunneling" (CDT) [6] to generate a ratchetlike motion. Unlike the majority of ratchets which depend on the presence of dissipation to operate, this motion results solely from the breaking of spatial and time symmetries in the system, and so preserves the quantum coherence of the system. Using this scheme, a pair of particles can be brought together and allowed to interactthereby becoming entangled-and then subsequently separated again to create entanglement between distinct spatial locations. We are thus able to both selectively move and entangle quantum particles using only the global operation of varying the parameters of the driving field, thus avoiding any need for the individual addressing of lattice sites.

Model. - The specific physical system that we consider consists of ultracold bosonic atoms, confined in a onedimensional optical lattice potential created by the superposition of counterpropagating laser beams. This form of confinement provides an extremely clean and controllable lattice potential, and in addition their high degree of isolation from the environment gives these systems rather long decoherence times, making them ideal for studying quantum coherent phenomena.
The single-particle dynamics of the system can be described extremely well by the Hamiltonian [7]

$$
H=\sum_{\langle i, j\rangle}\left[J_{i j} a_{i}^{\dagger} a_{j}+\text { H.c. }\right]+K \sin \omega t \sum_{i} x_{i} n_{i},
$$

where $a_{i} / a_{i}^{\dagger}$ are the standard bosonic destruction (creation) operators and the tunneling amplitudes $J_{i, j}$ connect nearest-neighbor sites $(i, j)$. Without loss of generality we shall henceforth take $J_{i j}=J$. The amplitude and frequency of the time-dependent driving field are described by the parameters $K$ and $\omega$, and $x_{i}$ is the spatial location of the $i$ th lattice site. This form of linear potential has already been used in cold atom experiments [8] to induce CDT, and can be straightforwardly implemented in an optical lattice by introducing a periodic phase modulation to one of the laser fields providing the standing wave potential.

Since the Hamiltonian (1) is invariant under discrete translations in time of the drive-period, $H(t)=$ $H(t+n T)$, the Floquet theorem allows us to write solutions of the Schrödinger equation as $\left|\phi_{n}(t)\right\rangle=\left|u_{n}(t)\right\rangle \times$ $\exp \left[-i t \epsilon_{n}\right]$, where $\left|u_{n}(t)\right\rangle$ is a $T$-periodic function called the Floquet function, and $\epsilon_{n}$ is termed the quasienergy. When two quasienergies approach degeneracy, the time scale to observe tunneling between the associated Floquet states diverges, and accordingly the tunneling between them appears suppressed. In the limit of high frequency (when $\omega$ is the dominant energy scale of the problem) it may be indeed shown [6,9-11] that the driven system behaves like the undriven one, but with renormalized tunneling amplitudes. For sinusoidal driving this renormalization takes the form $J_{\text {eff }}=J \mathcal{J}_{0}(K x / \omega)$, where $\mathcal{J}_{0}$ is the zeroth Bessel function of the first kind and $x$ is the intersite separation. Thus when $K x / \omega$ is equal to a zero of $\mathcal{J}_{0}$ the system's tunneling dynamics are frozen, producing CDT.

This way of regulating the tunneling between sites has been recently proposed to control the Mott-insulator transition in Bose-Einstein condensates $[12,13]$. To obtain a ratchet effect, however, it is necessary to distinguish between motion to the left and motion to the right. This may be achieved by noting that the argument of the Bessel function depends on the potential difference between 
neighboring sites, and thus on their spatial separation. Accordingly we consider a bipartite lattice of the form $A B A B A B$, as shown in Fig. 1, in which the $A B$ separation is not equal to that between $B A$. One possible realization of this would be a chain of coupled double-well potentials [14]. Consider placing a single particle in the center of this lattice [Fig. 1(a)]. In the absence of a driving field it will rapidly disperse by tunneling to both its neighbors. If, however, the system is driven by a high-frequency sinusoidal potential such that $\mathcal{J}_{0}\left(K x_{1} / \omega\right)=0$, then tunneling between sites separated by $x_{1}$ is destroyed and the lattice divides into a set of disconnected dimers $(A B)(A B)(A B)$. In this case the particle is unable to spread over the lattice, and is restricted to making a Rabi oscillation [Fig. 1(b)] between its initial location and its neighbor to the right. The frequency of this oscillation is determined by the value of the renormalized tunneling for this process $J_{\text {eff }}=$ $J \mathcal{J}_{0}\left(K x_{2} / \omega\right)$, which will in general be nonzero. Conversely, if the parameters of the driving field are chosen such that $\mathcal{J}_{0}\left(K x_{2} / \omega\right)=0$, then the lattice dimerizes as $(B A)(B A)(B A)$ as shown in Fig. 1(c), and the Rabi oscillation will occur between the initial site and its neighbor to the left.

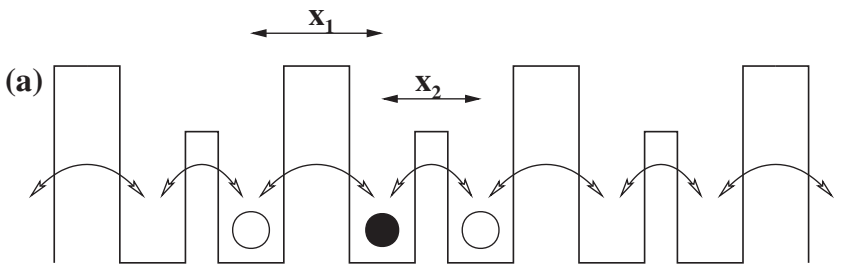

(b)

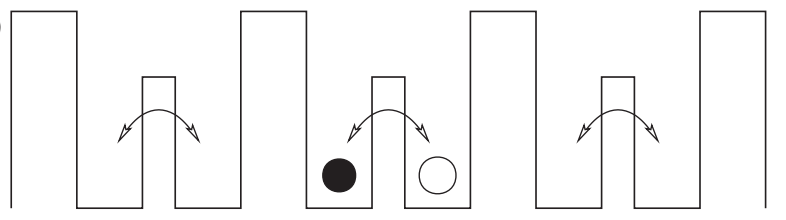

(c)

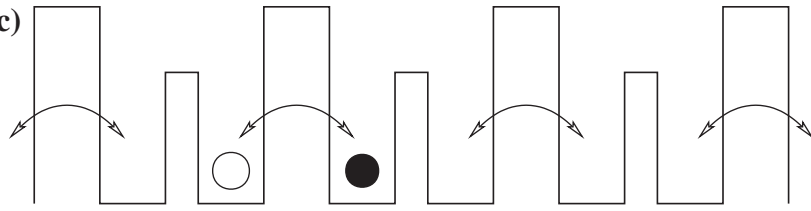

FIG. 1. We consider a bipartite lattice with two spacings: $x_{1}$ and $x_{2}$. Permitted tunneling processes are shown by the arrows. (a) In the absence of a driving field, a particle initialized in a given lattice site (the filled circle) can tunnel to either of its neighbors (empty circles). From there it can spread over the entire lattice. (b) If the lattice is sinusoidally driven with a field such that $\mathcal{J}_{0}\left(K x_{1} / \omega\right)=0$ then the tunneling processes between sites separated by $x_{1}$ are suppressed, and the particle can only tunnel to its right neighbor. (c) Conversely, if the driving field satisfies $\mathcal{J}_{0}\left(K x_{2} / \omega\right)=0$, then tunneling is only permitted between sites separated by $x_{1}$, and the particle can only tunnel to its left neighbor.
Results. - To verify this effect, we show in Fig. 2(a) the results of a numerical simulation of a single particle in a 16 -site system with $x_{1}=1$ and $x_{2}=0.75$. The frequency of the driving is set to a high value of $\omega=32 J$ to ensure that the system is in the high-frequency regime, while its amplitude satisfies $K x_{1} / \omega=2.4048$ - the first zero of $\mathcal{J}_{0}$. As the analysis predicts, the particle indeed simply oscillates between its initial location and one of its neighbors (that separated by a distance of $x_{2}$ ), since tunneling between sites separated by $x_{1}$ has been suppressed.

The crispness of the Rabi oscillation immediately suggests a scheme to produce directed motion. If we denote the period of this oscillation by $T_{2}$, then at $t=T_{2} / 2$ the particle has completely tunneled from its initial location $i$ to its neighbor $i+1$. If at this time the parameters of the field are altered so that $K x_{2} / \omega=2.4048$, then this tunneling process is suppressed and the particle instead begins to make a Rabi oscillation with period $T_{1}$ between sites $i+1$ and $i+2$. When a time interval of $T_{1} / 2$ has elapsed the particle has completely tunneled to site $i+2$. The driving
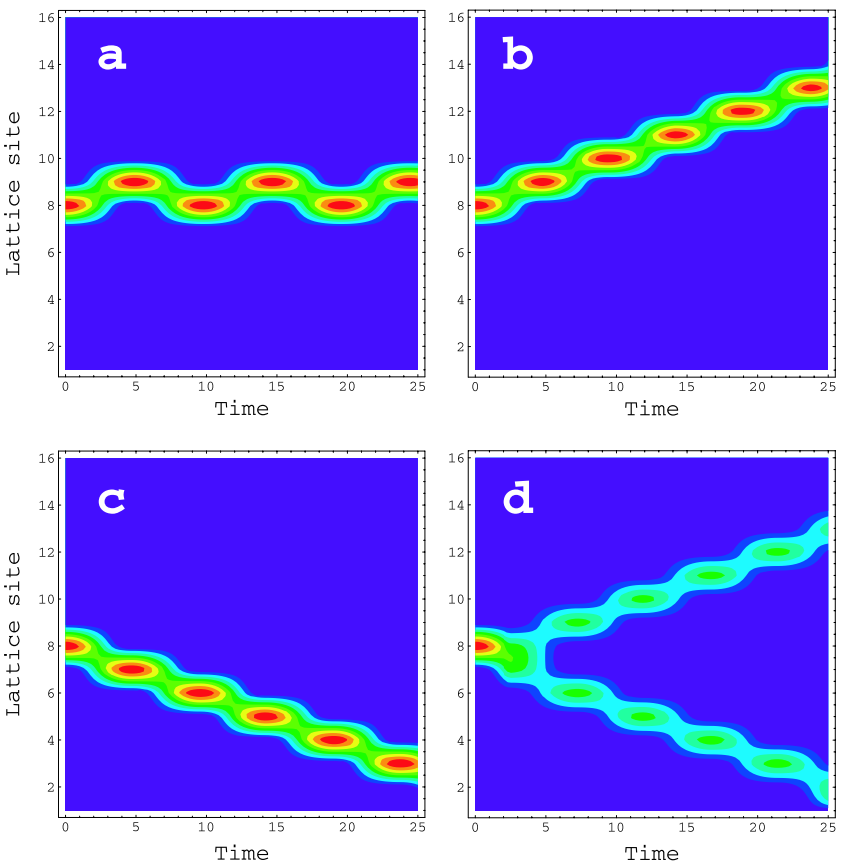

FIG. 2 (color online). Simulation of a single boson in a 16-site system. (a) Under a periodic driving field of amplitude $K x_{1} / \omega=$ 2.4048 we obtain the situation illustrated in Fig. 1(b) - tunneling between sites separated by $x_{1}$ is suppressed. Consequently the particle makes a Rabi oscillation to just one of its neighbors. (b) Under the driving field shown in Fig. 3 the $x_{1}$ and $x_{2}$ tunneling processes are periodically opened and closed, producing a ratchetlike motion of the particle. (c) Interchanging the order of the modulation of the driving field produces motion in the opposite direction. (d) Shortening the duration of the initial modulation means that the first tunneling process will be incomplete. If the initial modulation time is halved the particle splits into two equal parts, and under the driving field each part propagates in different directions. 
field can then be switched back to its original values and the procedure repeated. This has the effect of stepping the particle through the lattice in a sequence of discrete moves.

An example of a driving field that can produce this effect is shown schematically in Fig. 3. It can be thought of as a high-frequency "carrier wave" whose amplitude is modulated by a squarewave envelope. The lower amplitude segment suppresses tunneling between sites separated by $x_{1}$ and has a duration such that the particle tunnels exactly to its other neighbor (separated by $x_{2}$ ): the reverse is true for the higher amplitude segments. Figure 2(b) shows the response of the single-particle system to this field. Instead of the two-site Rabi oscillation seen previously, the particle now advances to the left in a series of well-defined steps. Conversely, if the order of the modulation is interchanged, the particle will propagate solely to the right as shown in Fig. 2(c). It is interesting to note that the direction of the particle's propagation also depends on which site of the double well it is initialized; particles started in the left well will move in the opposite direction to those placed in the right. The direction of motion thus depends on both the parity of the lattice site and the order of modulation, in a way not seen in standard dissipative ratchets. This flexibility requires, however, excellent control over the localization of the initial state.

We have so far considered the extreme cases in which propagation occurs in only one direction. If, however, the duration of the initial modulation is not exactly half a Rabiperiod, the initial tunneling process will not be complete. Consequently, the particle will divide into two parts, and under the subsequent influence of the driving field one part will propagate to the left while the other moves to the right. In Fig. 2(d) we show that if the initial modulation has a duration of $T_{2} / 4$ the particle splits in half. In this way, the

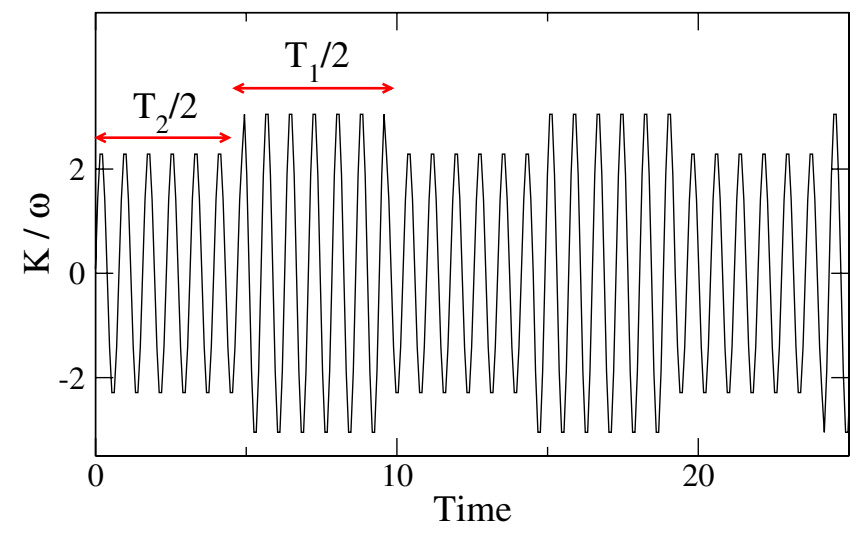

FIG. 3 (color online). The driving field producing the ratchetlike motion seen in Fig. 2 consists of a high-frequency sinusoidal oscillation with a modulated amplitude. The two values of the modulation satisfy $K / \omega=2.4048 / x_{i}$, and act to suppress the tunneling between sites separated by distances of $x_{i}$, respectively. The time intervals $T_{1}$ and $T_{2}$ are the Rabi periods for the renormalized tunneling processes between sites separated by $x_{1}$ and $x_{2}$. driving field can not only be used to control the motion of a particle, but also as a quantum beam splitter to divide a particle into a given superposition of left and right propagating components.

We have so far just considered single-particle manipulation. However, the ability to control the intersite tunneling also enables us to entangle particles and thereby realize quantum gates [15]. To demonstrate this, we first consider a two-site model occupied by two particles ( $a$ and $b$ ) that are distinguishable. This can be realized, for example, by using bosonic atoms $[4,7]$ with two different internal states [16]. For simplicity, we model the interaction between the particles as a Hubbard interaction

$$
H_{I}=\frac{U}{2} \sum_{i} n_{i}\left(n_{i}-1\right)
$$

where $U$ sets the interaction strength and $n_{i}=n_{i}^{a}+n_{i}^{b}$ gives the total number of bosons occupying site $i$. The dynamics of this system is governed by the interplay between the kinetic energy and the interaction, and consequently can exhibit a rather complicated time evolution. If, however, $U$ is much larger than the tunneling amplitude, the ground state of the system will then approximately consist of each site holding one particle, from which the doubly occupied states will be separated by an energy gap of $\sim U$. In this case canonical perturbation theory can be applied to eliminate the higher energy states, with the result that the Hubbard interaction maps to an effective Heisenberg term, with exchange constant given by $J_{H} \simeq$ $4 J_{\text {eff }}^{2} / U$. This mapping considerably simplifies analysis of the system's dynamics, and reveals that if the system is initialized in the state $|a, b\rangle$, the two particles swap positions after a time-interval $t=\pi / J_{H}$, while after $t_{S}=$ $\pi / 2 J_{H}$ the maximally entangled state $(|a, b\rangle+$ $i|b, a\rangle) / \sqrt{2}$ is produced. Applying the Heisenberg interaction for a duration of $t_{S}$ thus realizes the $\sqrt{\mathrm{SWAP}}$ operation.

In Fig. 4 we show the time evolution of a 10-site lattice, initialized with a boson of type $a$ in the first site, and a boson of type $b$ in the last. To ensure the validity of the mapping to the Heisenberg interaction, we require a high value for $U$. However, the time required to entangle the particles is proportional to $U$, and so to complete as many quantum gate operations as possible within the system's decoherence time we would like to take $U$ to be as small as possible. We thus consider an intermediate value of $U=$ $4 J$, and as before use a driving frequency of $\omega=32 \mathrm{~J}$ to place the system in the high-frequency regime. Under the influence of the driving field the two bosons are progressively stepped through the lattice towards the two central sites, whereupon the amplitude of the driving is held at a constant value to retain the two particles there. After being held there for a time interval of $t_{S}$, the particles are then separated and returned to the first and last lattice sites.

From Fig. 4(a) it can be clearly seen that the particles remain highly localized in space, and accordingly the 

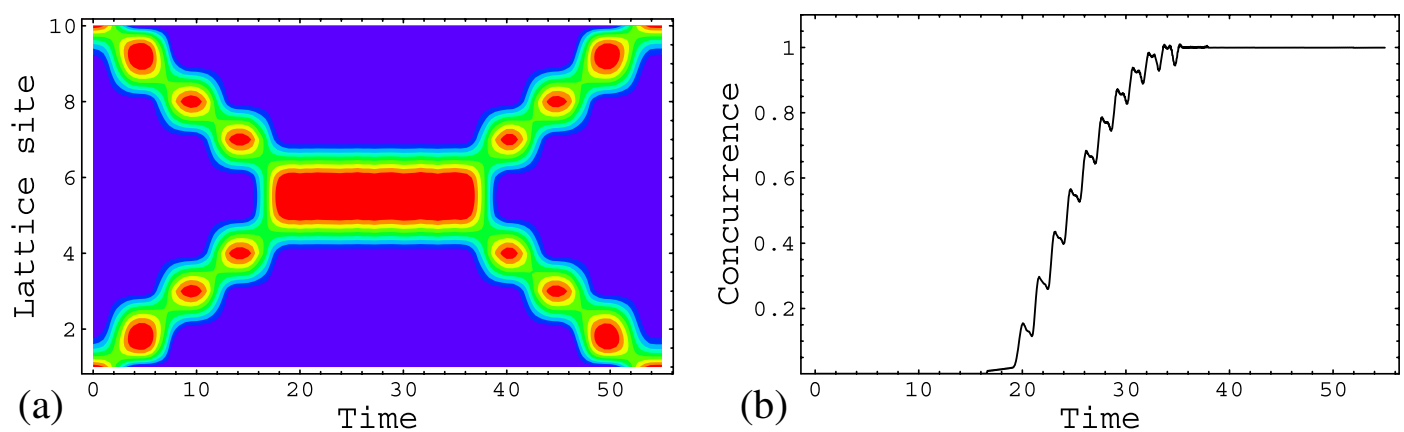

FIG. 4 (color online). (a) Time development of the system initialized with one boson in the first lattice site, and another in the final site. Under the influence of the driving field they are moved toward the center of the array, held there for a time-interval $t_{S}$, and then restored to their original positions. (b) The pairwise concurrence present in the system. Initially this is zero since the initial state is not entangled, but rises as the two bosons interact (via Heisenberg exchange). The interval over which the interaction occurs is chosen to maximize the concurrence, by producing a maximally entangled state.

probability distribution is generally peaked at two sites. This permits a simple measurement of the entanglement present in the system by projecting the wave function onto just these two sites, and then evaluating the two-particle concurrence $C(t)$ [17], plotted in Fig. 4(b). Initially the concurrence is zero since the particles have not interacted, and so the two-particle wave function is factorizable. This remains true as the particles approach each other, until they reach the two central sites. Driven by the Heisenberg interaction, the concurrence then rises from zero following the approximate time-dependence $C(t)=\left|\sin J_{H} t\right|$. The high-frequency ripples visible in this quantity arise from the influence of the higher energy states: if $U$ is increased these ripples will be quenched, but equally $J_{H}$ will be reduced, and so the time scale for entanglement to occur will increase. When the particles are separated the degree of entanglement remains "frozen-in" at its final value. It is thus possible to generate any desired degree of entanglement by controlling the period during which the particles interact. When this period is equal to $t_{S}$, as shown in Fig. 4, the entanglement is maximized, and the final state of the system thus represents a mesoscopically separated, maximally entangled two-particle state.

Conclusions. - In summary, we have shown how a periodic driving field can induce a novel ratchetlike motion, which can be employed to selectively guide and divide particles. In addition the interaction between the particles can be used to entangle them, and thus realize fundamental two-qubit quantum gates, such as $\sqrt{\text { SWAP. }}$. In this work we have just considered a one-dimensional geometry, but an attractive aspect of optical lattices is the possibility of generating a higher-dimensional [14] lattice potentials, which would allow parallel processing of qubits, and thus be used to greatly enhance their error tolerance. Finally, although we have specifically considered a system of ultracold bosons, the method we have described could equally be applied to optically confined fermionic atoms [18], or to electronic transport in systems such as coupled quantum dots [19] or molecular wires [20].

This research was supported by the EPSRC. The author acknowledges the hospitality of the University of Edinburgh where this work was completed, and thanks Sougato Bose for stimulating conversations.

[1] D. Loss and D. P. DiVincenzo, Phys. Rev. A 57, 120 (1998).

[2] L. B. Ioffe et al., Nature (London) 398, 679 (1999).

[3] S. Bose, Phys. Rev. Lett. 91, 207901 (2003).

[4] D. Jaksch et al., Phys. Rev. Lett. 82, 1975 (1999).

[5] G. K. Brennen et al., Phys. Rev. Lett. 82, 1060 (1999).

[6] F. Grossmann et al., Phys. Rev. Lett. 67, 516 (1991).

[7] D. Jaksch et al., Phys. Rev. Lett. 81, 3108 (1998).

[8] K. W. Madison et al., Phys. Rev. Lett. 81, 5093 (1998); H. Lignier et al., arXiv:0707.0403.

[9] D. H. Dunlap and V. M. Kenkre, Phys. Rev. B 34, 3625 (1986).

[10] M. Holthaus, Phys. Rev. Lett. 69, 351 (1992).

[11] C. E. Creffield, Phys. Rev. B 67, 165301 (2003).

[12] A. Eckardt, C. Weiss, and M. Holthaus, Phys. Rev. Lett. 95, 260404 (2005).

[13] C.E. Creffield and T. S. Monteiro, Phys. Rev. Lett. 96, 210403 (2006).

[14] J. Sebby-Strabley et al., Phys. Rev. A 73, 033605 (2006).

[15] N. Teichmann and C. Weiss, Europhys. Lett. 78, 10009 (2007).

[16] It is important to note, however, that the two species of atoms should see the same optical lattice potential.

[17] W. K. Wootters, Phys. Rev. Lett. 80, 2245 (1998).

[18] M. Köhl et al., Phys. Rev. Lett. 94, 080403 (2005).

[19] C.E. Creffield and G. Platero, Phys. Rev. B 69, 165312 (2004).

[20] J. Lehmann et al., Phys. Rev. Lett. 88, 228305 (2002). 